\title{
Correction to: New Generation Devices for Transcatheter Aortic Valve Implantation
}

Laura lop and Gino Gerosa

\section{Correction to: A. Giordano et al. (eds.), Transcatheter Aortic Valve Implantation, https://doi.org/10.1007/978-3-030-05912-5_45}

The first and last name of the authors, Laura Iop and Gino Gerosa were exchanged in the original version. This has been corrected now.

The updated online version of this chapter can be found at https://doi.org/10.1007/978-3-030-05912-5_45 